\title{
Journal watch: diagnostic nanoparticles
}

First draft submitted: 21 September 2016; Accepted for publication: 26 September 2016; Published online: 17 January 2017

Keywords: atherosclerosis $\bullet$ circulating tumor cells detection • imaging and diagnostic nanosystems $\bullet \mathrm{NIRF} \bullet \mathrm{PET} \bullet$ photoacoustic cancer imaging $\bullet$ Raman imaging

\section{Magnetic nanowires for} ultrasensitive detection \& isolation of rare cancer cells

Evaluation of: Hong W, Lee S, Chang HJ, Lee ES, Cho Y. Multifunctional

magnetic nanowires: a novel breakthrough for ultrasensitive detection and isolation of rare cancer cells from nonmetastatic early breast cancer patients using small volumes of blood. Biomaterials 106, 78-86 (2016).

Since metastatic disease is the most common cause of death in cancer patients [1], an early identification of circulating tumor cells (CTCs) would enable a great diagnostic and prognostic advancement. Numerous approaches have been proposed to detect CTCs, including density gradient- and sizebased filtration or immuno-magnetic selection $[2,3]$, but among their drawback are false negative or false positive results due to unspecific binding [4]. Moreover, CTCs are very rare, with roughly 1-10 CTCs being found in $1 \mathrm{ml}$ of blood in a patient with metastatic cancer. New sensitive methods to detect and isolate CTCs are thus urgently needed, particularly for patients with early cancer stages.

To address this need, Hong et al. developed multifunctional magnetic nanowires (NW) composed of magnetic nanoparticle-doped polypyrrole, bound with a mixture of five antibodies via ss-biotin molecules (Ab-PpyNW). The authors first confirmed the efficacy of AbPpyNW to capture different types of cancer cells in healthy blood samples spiked with specific numbers of tumor cell line-derived cells. After optimizing the capture conditions of Ab-PpyNW, peripheral blood of 29 nonmetastatic early-stage breast cancer patients was tested and compared with 18 healthy donors. While $5-10 \mathrm{ml}$ of blood is currently necessary to identify CTCs, in this novel approach, $250 \mu \mathrm{l}$ were sufficient to detect CTCs, whereby sensitivity increased with higher volume. A significant number of single CTCs and CTC clusters were identified in all blood samples of cancer patients analyzed, according to the stage and the received therapy, whereas in 16 of 18 healthy donors no CTCs were identified. In the remaining two healthy donors, $1-2$ cells per $1 \mathrm{ml}$ of blood were detected. The captured cells could easily be removed from Ab-PpyNW by glutathione-mediated release, allowing further immunohistochemical analyses. The authors have additionally developed a colorimetric technique to quickly and reliably identify the presence of CTCs in blood by adding tetramethylbenzidine to the cell suspension, which generates a color signal proportional to the number of captured cancer cells.

Multifunctional magnetic NW-based CTC isolation technique thus enables precise identification as well as ultrasensitive detection of various CTC types. By capturing CTCs from a small volume of blood, this method should advance treatment, monitoring and prognosis in numerous cancer patients.

- Written by M Pöttler
Marina Pöttler', Iwona Cicha', Stefan Lyer', Christina Janko', Ralf P Friedrich ${ }^{1}$ \& Christoph Alexiou $*, 1$

'ENT-Department, Section of Experimental Oncology \& Nanomedicine (SEON), Else Kröner-Fresenius-StiftungProfessorship for Nanomedicine, University Hospital Erlangen, Glueckstr. 10a, 91054 Erlangen, Germany *Author for correspondence: c.alexiou@web.de 


\section{HDL nanoparticles for PET imaging of atherosclerosis \\ Pérez-Medina C, Binderup T, Lobatto ME et al. In vivo PET imaging of HDL in multiple atherosclerosis models. JACC Cardiovasc. Imaging 9(8), 950-961 (2016).}

Atherosclerotic plaque rupture is responsible for the majority of deaths in the world. However, most of the current diagnostic modalities used for clinical atherosclerosis imaging provide limited information about plaque rupture risk or the activity of inflammatory processes. Due to its superb sensitivity, positron emission tomography (PET), an imaging technique which allows detection of nanomolar radiotracer concentrations, offers the possibility of molecular imaging of plaque progression [5].

In their paper, Pérez-Medina et al. utilized zirconium-89 $\left({ }^{89} \mathrm{Zr}\right)$ and the chelator deferoxamine $\mathrm{B}$ to label the phospholipid or apolipoprotein A-I components of the high-density lipoprotein (HDL) particles. The resulting ${ }^{89} \mathrm{Zr}-\mathrm{AI}-\mathrm{HDL}$ and ${ }^{89} \mathrm{Zr}$-PL-HDL particles had size of native HDL $(8.5 \mathrm{~nm})$. As control, HDL particles conjugated with DiR, a near-infrared fluorescence dye were produced.

Following the detailed biodistribution studies, the authors demonstrated the accumulation of both particle types in the plaque-affected aortic regions in the $A p o E^{-1-}$ mice fed high fat diet by micro-PET/ CT and ex vivo near-infrared fluorescence, as well as in a rabbit model of balloon injury-induced aortic atherosclerosis by large animal PET/CT and PET/ MRI. Further, targeting of ${ }^{89} \mathrm{Zr}$-PL-HDL particles to the femoral plaques was confirmed by PET/CT in a porcine model of accelerated atherosclerosis induced by familial hypercholesterolemia and balloon injury. Independent of the radiolabel location on the particle, preferential uptake of the ${ }^{89} \mathrm{Zr}$-labeled HDL particles by plaque macrophages and monocytes was observed. Importantly, the labeling with ${ }^{89} \mathrm{Zr}$ did not affect the natural behavior and distribution of HDL. In contrast, oxidized HDL particles $\left.{ }^{89} \mathrm{Zr}-\mathrm{AI}-\mathrm{HDL}^{\mathrm{Ox}}\right)$ showed a significantly faster blood clearance in both atherosclerotic and healthy rabbits, along with increased kidney uptake. Consequently, at day five postinjection, their accumulation in atherosclerosis-affected aortas was significantly lower than that of nonoxidized ${ }^{89} \mathrm{Zr}$-AIHDL particles.

Although the long half-life of ${ }^{89} \mathrm{Zr}$ may prevent its use as a radiotracer in humans, the potential applicability of those particles for long-term tracking of in vivo HDL behavior, but also for providing insight into HDL ability to identify high-risk plaques in preclinical models is high. Additionally, the use of the HDL-like particles as a drug delivery platform, proposed by the authors in the previous studies in mice [6] is now open to further development, as its capability of plaque targeting in models closer to human atherosclerosis has been confirmed. ${ }^{89} \mathrm{Zr}$-HDL particles could thus constitute an attractive tool for monitoring of drug delivery to the plaque.

- Written by I Cicha

\section{Photon roller coaster for enhancing near} infrared optical imaging of tissue

Evaluation of: Levy ES, Tajon CA, Bishof

TS et al. Energy-looping nanoparticles:

harnessing excited-state absorption for

deep-tissue imaging. ACS Nano Article ASAP, doi:10.1021/acsnano.6b03288,

Web-publication: 2016-10-07.

Due to low scattering and adsorption of near infrared light (NIR) photons by tissue, for imaging purposes NIR is superior to visible light and enables imaging at a micro-scale resolution through millimeters of tissue. In their recent paper, Levy et al. developed a new class of materials for enhanced NIR-deep-tissue-imaging by doping $\beta-\mathrm{NaYF}_{4}$-nanoparticles with lanthanides. These particles, called energy-looping nanoparticles, exploit a positive feedback loop of intermediate excited states resulting in a dramatically increased absorption of photons upon excitation at wavelengths in the NIRII window. Additionally, this process can also lead to an upconversion of the emitted photons, which do not interfere with the dopants themselves.

To identify the lanthanide dopant compositions exhibiting the desired properties, the authors first used a high-throughput computational screening. The screening was done with materials excitable at $1064 \mathrm{~nm}$, which allow the use of common lasers for the experiments. $\mathrm{Tm}^{3+}$ exhibited the highest rates of upconverted luminescence, with an emission at $800 \mathrm{~nm}$, and could not be enhanced by co-doping with other ions. This was unexpected, since $\mathrm{Tm}^{3+}$ is not known to have an absorption peak at $1064 \mathrm{~nm}$. The experimental tests on a library of $\mathrm{NaYF}_{4}$-nanoparticles doped with different amounts of $\mathrm{Tm}^{3+}$ revealed that the energy used at $1064 \mathrm{~nm}$ in standard spectrophotometers is too low to produce the upconversion. In contrast, the laser of a confocal microscope was able to induce the desired effect in the range predicted by the simulations. Following a deep mechanistic study of the looping effects of, the authors performed the analyses of their imaging properties (excitation: $1064 \mathrm{~nm}$; emission: $800 \mathrm{~nm}$ ) on $1 \mathrm{~mm}$ phantoms and mouse brain tissue. Using $5 \mu \mathrm{m}$ polystyrene (PS) beads loaded with the $\mathrm{Tm}^{3+}$-energylooping nanoparticles, the authors showed that this new class of material is superior to the current stateof-the-art upconversion nanoparticles (excitation: 
$784 \mathrm{~nm}$ ) being clearly visualized through the tissue with a resolution of approximately $2 \mu \mathrm{m}$.

The authors concluded that this new class of dyes shows an improved resolution at a tissue depth of 1 $\mathrm{mm}$, which is comparable to the state-of-the-art multiphoton techniques, but with significantly lower costs and infrastructure. It will be very interesting to follow, if the computational screening can discover further, perhaps even more potent dyes of this class, and how this will influence the analysis of biological mechanisms in living tissue.

- Written by S Lyer \& C Alexiou

\section{Intraoperative visualization of liver tumors using RAMAN scattering nanoparticles} Evaluation of: Andreou C, Neuschmelting V, Tschaharganeh $\mathrm{D}$ et al. Imaging of liver tumors using surface-enhanced Raman scattering nanoparticles. ACS Nano 10, 5015-5026 (2016). Surgery remains the gold standard and often the only curative option in liver cancer. For complete surgical removal of a tumor and subsequent therapeutic success, a precise visualization of the tumor during surgery is crucial. Conventionally, liver tumors are detected by MRI, CT, PET or ultrasound, the techniques which have limitations in terms of intraoperative imaging and sensitivity, or are accompanied by ionizing radiation or radioactive imaging agents [7]. To address the need of a new precise intraoperative imaging technique, Andreou et al. explored the utility of surface-enhanced Raman scattering nanoparticles (SERS-NP) for liver imaging. To produce SERS-NPs, a reporter molecule with a specific Raman signature was adsorbed on a gold core and encapsulated in a silica shell, resulting in a final NP size of 108 $\mathrm{nm}$ and a zeta potential ( $\mathrm{pH} 7.1)$ of $-46.9 \mathrm{mV}[8,9]$. Based on the respective Raman reporter dye, the NP produced distinct stable spectra in serum for $24 \mathrm{~h}$.

Being rich in phagocytic cells of the reticuloendothelial system, normal liver tissue rapidly takes up nanoparticles. When phagocytic cells are replaced by tumor cells, nanoparticle uptake decreases dramatically and this phenomenon can be utilized for cancer imaging. After intravenous injection in mice, SERSNP were rapidly cleared by reticuloendothelial system. Real-time monitoring of the Raman signal showed a maximum intensity in the blood immediately after nanoparticle injection, with steady decrease due to sequestration of nanoparticles from the circulation. Accordingly, the Raman signal in the liver increased, reaching plateau within several minutes and after $12 \mathrm{~h}$, tissue homogenates of livers and spleens showed a characteristic Raman signature.

To compare the localization of the nanoparticles in healthy liver and tumor tissue, analysis of the Raman signature was supported by a direct classical leastsquares model, so that normal liver tissue (Raman reference spectrum), background and cancer tissue could be clearly discriminated by use of a color scheme. Mouse models of hepatocellular carcinoma (Myc-driven) and histiocytic sarcoma (Ink4A/Arf $f^{\prime}$ ) were employed to prove the in vivo imaging capacity of the SERS-NP. In both models, intraoperative Raman imaging of surgically exposed livers enabled the successful detection of microscopic cancerous lesions $(250 \mu \mathrm{m})$, which were not detectable by MRI, visual inspection or palpation, but were confirmed by subsequent histology. Moreover, SERS-NP detected tumors with more sensitivity and specificity than indocyanine green (ICG), an established contrast agent for fluorescence imaging of liver tumors [10], due to varying tumor-to-background ratios and photobleaching of the fluorescence dye. Remarkably, SERS-NP reached a factor 40 difference in accumulation between tumor and healthy liver.

In conclusion, NP-enhanced Raman imaging can be applied for high-accuracy detection of liver tumors in mice. Although Raman imaging devices for clinical use are still under development and the regulatory approval of NP-based imaging agents might be challenging, this in vivo proof-of-principle study shows the clinical potential of this technique.

- Written by C Janko

\section{Nanoparticle-based photoacoustic pH imaging \& pH-responsive tumor therapy} Evaluation of: Chen Q, Liu X, Zeng J, Cheng Z, Liu Z. Albumin-NIR dye self-assembled nanoparticles for photoacoustic $\mathrm{pH}$ imaging and $\mathrm{pH}$-responsive photothermal therapy effective for large tumors. Biomaterials 98 , 23-30 (2016).

The microenvironment of solid tumors and healthy tissue are strongly different. In contrast to normal tissue, the hypoxia-induced neovasculature of growing tumors is often insufficient, resulting in poor perfusion, elevated interstitial fluid pressure, nutrient deficiency and severe acidity [11]. In spite of that, tumor cells can adapt to these hostile conditions, which may lead to therapeutic resistance and enhanced malignant behavior [12]. In recent years, great efforts were made to target the specific tumor environment $[12,13]$, in particular the acidic extracellular $\mathrm{pH}$, which has been found suitable for targeted therapy and imaging using $\mathrm{pH}$-responsive systems [14,15].

In their recent paper, Chen et al. have developed $\mathrm{pH}$-responsive self-assembled nanoparticles by adsorption of NIR dye croconine (Croc) onto human serum albumin (HSA) via hydrophobic interaction. The HSA-Croc nanoparticles were purified by centrifuga- 
tion and ultrafiltration and characterized by transmission electron microscopy, UV-vis-NIR spectrophotometry and dynamic light scattering. The highly stable, monodispersed $100 \mathrm{~nm}$ HSA-Croc particles revealed low Croc release and negligible cytotoxicity even at high particle concentrations. Importantly, the absorption peaks of HSA-Croc at $680 \mathrm{~nm}$ and $790 \mathrm{~nm}$ exhibited strong and reversible $\mathrm{pH}$-dependence especially in the $\mathrm{pH}$-range of acidic tumor microenvironment. Thus, IR thermal images and temperature measurements of HSA-Croc solutions under the irradiation of an NIR laser showed an enhanced photothermal effect at values below $\mathrm{pH} 7$.

This dual-wavelength ratiometric photoacoustic imaging and $\mathrm{pH}$-responsive photothermal effect was also demonstrated in 4T1 tumor-bearing mice. An increased photothermal heating of tumor tissue and strongly reduced nonspecific photothermal heating of normal tissues were observed after intravenous administration of HSA-Croc and exposure to a NIR laser. As a result, the $4 \mathrm{~T} 1$ tumors were completely eliminated in presence of HSA-Croc. In control mice, treated with non-pH-responsive indocyanine loaded particles (HSA-ICG), three of five tumors showed only partial reduction and continued to grow at later time points.

\section{References}

1 Wittekind C, Neid M. Cancer invasion and metastasis. Oncology 69(Suppl. 1), 14-16 (2005).

2 Lee $\mathrm{HJ}$, Oh JH, Oh JM et al. Efficient isolation and accurate in situ analysis of circulating tumor cells using detachable beads and a high-pore-density filter. Angew. Chem. Int. Ed. Engl. 52, 8337-8340 (2013).

3 Yoon HJ, Kim TH, Zhang Z et al. Sensitive capture of circulating tumour cells by functionalized graphene oxide nanosheets. Nat. Nanotechnol. 8, 735-741 (2013).

4 Lianidou ES, Markou A. Circulating tumor cells in breast cancer: detection systems, molecular characterization, and future challenges. Clin. Chem. 57, 1242-1255 (2011).

5 Joseph P, Tawakol A. Imaging atherosclerosis with positron emission tomography. Eur. Heart J. pii:ehw147 (2016) (Epub ahead of print).

6 Duivenvoorden R, Tang J, Cormode DP et al. A statinloaded reconstituted high-density lipoprotein nanoparticle inhibits atherosclerotic plaque inflammation. Nat. Commun. 5, 3065 (2014)

7 Bonanni L, Carino ND, Deshpande R et al. A comparison of diagnostic imaging modalities for colorectal liver metastases. Eur. J. Surg. Onc. 40, 545-550 (2014).

8 Harmsen S, Huang RM, Wall MA et al. Surfaceenhanced resonance Raman scattering nanostars for high-
Due to the more acidic microenvironment of the tumor core, the resulting photoacoustic signals were much higher in HSA-Croc-injected mice than in HSA-ICGtreated controls. Consequently, the increased NIR absorbance in the tumor core led to the more effective photothermal heating of large tumors after HSA-Croc treatment.

Taken together, an intravenous injection of HSACroc enables the real-time visualization and diagnosis of changes in tumor $\mathrm{pH}$ by dual wavelength ratiometric photoacoustic imaging. This study represents a promising novel approach to $\mathrm{pH}$-responsive photothermal therapy by targeting the acidic microenvironment of the tumors.

- Written by RP Friedrich

\section{Financial \& competing interests disclosure}

The authors have no relevant affiliations or financial involvement with any organization or entity with a financial interest in or financial conflict with the subject matter or materials discussed in the manuscript. This includes employment, consultancies, honoraria, stock ownership or options, expert testimony, grants or patents received or pending, or royalties.

No writing assistance was utilized in the production of this manuscript.

precision cancer imaging. Sci. Transl. Med.7(271), 271ra7 (2015).

9 Harmsen S, Bedics MA, Wall MA, Huang RM, Detty MR, Kircher MF. Rational design of a chalcogenopyrylium-based surface-enhanced resonance Raman scattering nanoprobe with attomolar sensitivity. Nat. Commun. 6, 6570 (2015).

10 Ishizawa T, Masuda K, Urano Y et al. Mechanistic background and clinical applications of indocyanine green fluorescence imaging of hepatocellular carcinoma. Ann. Surg. Oncol. 21, 440-448 (2014).

11 Horsman MR, Vaupel P. Pathophysiological basis for the formation of the tumor microenvironment. Front. Oncol. 6, 66 (2016).

12 Cavazos DA, Brenner AJ. Hypoxia in astrocytic tumors and implications for therapy. Neurobiol. Dis. 85, 227-233 (2016).

13 Paolicchi E, Gemignani F, Krstic-Demonacos M, Dedhar S, Mutti L, Landi S. Targeting hypoxic response for cancer therapy. Oncotarget 7, 13464-13478 (2016).

14 Song CW, Griffin R, Park HJ. Influence of tumor $\mathrm{pH}$ on therapeutic response. In: Cancer Drug Discovery and Development: Cancer Drug Resistance. Teicher B (Ed.). Humana Press Inc., Totowa, NJ (2007).

15 Liu J, Huang Y, Kumar A et al. $\mathrm{pH}$-sensitive nano-systems for drug delivery in cancer therapy. Biotechnol. Adv. 32, 693-710 (2014). 